\title{
Phase Center Stable Ultra Wide-Band Antenna for EMC Field Sensing
}

\author{
S. Bruni ${ }^{1}$, A. Neto ${ }^{1}$, F. Marliani ${ }^{2}$ \\ ${ }^{1}$ TNO - Defence, Security and Safety, Oude Waalsdorperweg 63, 2597AK The Hague, The Netherlands. \\ E-Mail: simona.bruni@tno.nl, andrea.neto@tno.nl \\ ${ }^{2}$ European Space Research and Technology Center (ESTEC), Noordwijk, The Netherlands. \\ E-Mail: Filippo.Marliani@esa.int
}

\begin{abstract}
Leaky lens antennas are linearly polarized directive antennas constituted by long slots etched on a ground plane that separates a dense dielectric, shaped to form a lens, from free space. These antennas are characterized by significant directivity, very low dispersion and they are suited to be integrated with the receive modules. These properties arise from the particular leaky wave radiation mechanism that these antennas exploit. In this contribution, the ultra-wide band version of the antenna described in [4] is presented.
\end{abstract}

\section{INTRODUCTION}

In the field of EMC, there is an increasing need for ultra-wide band field sensors. For the lower frequency ranges, solutions that have recently been proposed with success make use of Mach Zehnder (MZ) devices [1], [2]. These latter are optical devices composed of special dielectric materials sensible to minimal field intensities. They are typically connected to small dipole antennas, so that the incoming RF electric field produces a measurable alteration of the optical signal. This optical signal can then be transmitted via fibre optics and thus with extremely low losses and very little interference with the device under analysis. However, the dimensions of the dipole limit the higher operational frequency of such devices. When one tries to operate this sensor at higher frequencies, the dipole shows resonant behavior, thus the frequency independence is lost. For higher frequencies MZ sensors have been proposed [2] with some success up to $12 \mathrm{GHz}$. In a project supported by ESA-ESTEC [3] an investigation has been performed to see how the MZ principle of operation could be adopted in higher frequency ranges. In particular a new design of the sensor antenna has been outlined. It essentially uses a different implementation of the leaky lens antenna concept discussed in [4]: the slot line is folded on the two side of a properly shaped dielectric lens instead of being terminated by a short circuit. This provides for a further enlargement of the operational bandwidth. This antenna is then connected to a travelling wave Mach Zehnder device, showing that a significant improvement of the overall Antenna Coupling Factor of the sensor could be achieved with respect to using standard microwave antennas and entirely microwave chains. In particular the extension of the use of $\mathrm{MZ}$ sensors to the upper range $(10 \mathrm{GHz}$ to $100 \mathrm{GHz})$ should compensate for the non portability of the measurement equipment. Instead of connecting the antennas, used to probe the field, directly to the network analyzers via coaxial cables that present extremely high losses, one could connect them to the optical fibers via the MZ sensor. This solution allows a sensible reduction of the loss figure which is proportional to the distance from the equipment to the device under test. In the following this novel antenna concept is called Pyramid antenna.

\section{ANTENNA DESCRIPTIONS}

The UWB version of the leaky lens antenna concept is depicted in Fig. 1. The lens geometry is the composition of two leaky lens antennas [4]. The shape of each one of these dielectric lenses is obtained as union of an infinite set of cross section planes of truncated elliptical shape and decreasing dimension. Each of the ellipses contains a slot etched on a ground plane, in the lower focus. The eccentricity of the ellipse is $e=1 / \sqrt{\epsilon_{r}}$, with $\epsilon_{r}$ the dielectric constant of the lens. In correspondence of the joining of the two slots and of the two original lenses, the shape of each one of the lenses is altered in order to host a ground plane of width $g$, on $x-y$ plane, see the side view in the inset of Fig. 1. This ground plane hosts the Coplanar Waveguide (CPW) transmission line, that will feed the unique slot that extends under both lenses.

For the sake of convenience the origin of the reference system is in the center of the CPW ground plane. The values of the angles $\alpha_{t}$ and $\gamma_{L W}$ are only functions of the lens dielectric constant: $\gamma_{L W}=\cos ^{-1} \sqrt{\frac{\epsilon_{r 1}+\epsilon_{r 2}}{2 \epsilon_{r 2}}}$ and $\alpha=\frac{\pi}{2}-\gamma_{L W}$.

As explained in [5] leaky wave rays in the dense dielectric are originated from each point of a slot etched in a ground plane between two infinite homogeneous dielectrics. Also in the present case, even if the problem is not planar, rays emerge from the slot. For each of the elliptical cross section the chosen eccentricity implies 


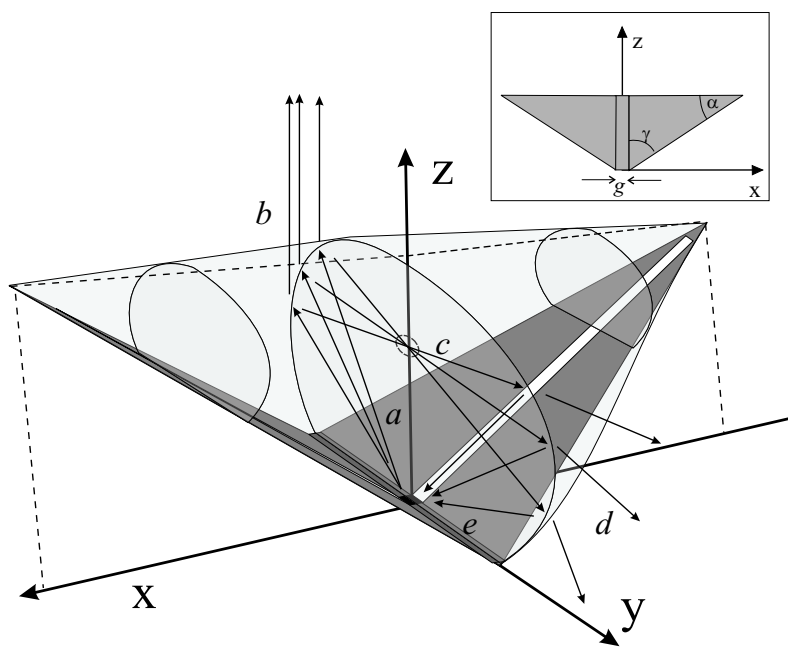

Fig. 1. Geometry of the Pyramid antenna with relevant rays in the central cross section. Inset: Side view.

the rays a), b), c), d), e) drawn in Fig. 1 for the central cross section. The different rays are: a) emanated from the slot (lower focus), b) transmitted after the first interface (focusing effect in the far field), c) reflected at the first interface, d) transmitted at the second interface (unfocused), e) reflected at the second interface and refocused toward the slot. The first transmitted rays, that carry most of the power due to small reflection at the lens-air interface, are all focused in one direction. Asymptotically, only doubly reflected rays, that already lost power in the first two transmissions, return back to the focus (slot line). This guarantees that the present lens simulates well the ideally infinite dielectric configuration characterized by very low frequency dispersivity. Note that the lenses are oriented in such a way that the rays generated in both arms of the unique radiating slot are focused, at least in first approximation, in broad side ( $z$-direction). The antenna is designed to be used in the band ranging from $4 \mathrm{GHz}$ to $40 \mathrm{GHz}$. In correspondence of the tip of the ground-plane, the shape is altered in order to form a small strip laying on the $x-y$ plane, see Fig. 1. This portion of the ground plane hosts the Coplanar Waveguide (CPW) transmission line with 50 Ohm impedance, feeding the slots that extends on both sides and generating two leaky waves, one along each arm.

The complete sensor will be integrated with a MZ interferometer, that transforms the received electric signal in an optical one. The MZ will be hosted in the CPW feeding line since most of the already existing MZ devices are based on travelling wave operation on CPW's. An antenna prototype was manufactured using ceramic material (TMM 3 from Rogers) for the lens, while the slot and CPW were etched from a copper layer deposited on the bottom face of the lens.

\section{Manufacturing AND MeAsurements}

A prototype of the Pyramid antenna was manufactured using TMM 3 (from Rogers) for the lens. The slot and CPW will be etched from a copper layer deposited on the bottom face of the lens itself. The final result is shown in Fig. 2, the lens length, height and width are $18 \mathrm{~cm} \times 10 \mathrm{~cm} \times 7 \mathrm{~cm}$ respectively. The CPW

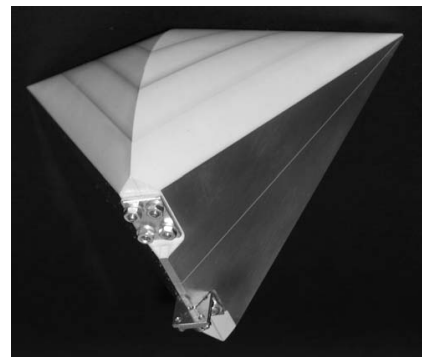

(a)

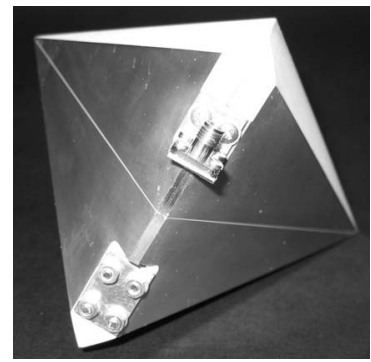

(b)

Fig. 2. Finals prototype: (a) lens; (b) feeding network.

line is as short as possible and reaches on one side of the radiating slot to the SK coaxial connectors. In this design, no air bridges have been included since the design is intrinsically balanced. In general, the leaky lens was meant to furnish an integrated receiver, thus the external coaxial connection could be avoided in a real system that would take benefit of the possible integration space at the side of the slot.

\section{A. Reflection Coefficient}

The first quality parameter of an UWB antenna is the impedance bandwidth. The simulated and measured reflection coefficients of the antenna when fed by a 50 $\mathrm{Ohm}$ transmission line are plotted in Fig. 3. The results are pertinent to simulations on an equivalent planar structure that could be represented using the commercial Method of Moments tool Ansoft Designer. The CPW port is located at $1 \mathrm{~cm}$ of distance from the CPW-slot to represent realistically the distance between the slot and the CPW-coaxial transition. The reflection coefficient is 


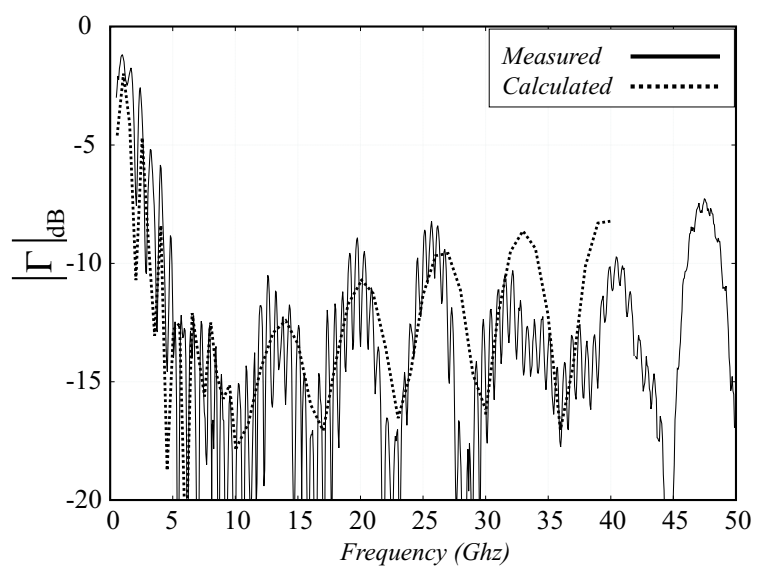

Fig. 3. Reflection coefficient simulated and measured at a CPW port located at distance of $10 \mathrm{~mm}$ from the CPW-slot intersection. The simulations are pertinent to an equivalent planar structure obtained using the commercial code Ansoft Designer.

below $9 \mathrm{~dB}$ for almost one decade of bandwidth (4-40 $\mathrm{GHz}$ ). For very low frequencies, the radiating slot is so short in terms of the wavelengths that some power reflected at the end-points alters the input impedance. The broader oscillations are due to the fact that the CPW line has a finite length and the input impedance varies over frequency.

\section{B. Radiation Pattern}

The radiation patterns have been calculated, for frequencies up to $10 \mathrm{GHz}$ using HFSS. Fig. 4 shows the co-polar radiation patterns in the $H$-plane for different frequencies: $4 \mathrm{GHz}$ (dashed line), $5 \mathrm{GHz}$ (dotted line), $10 \mathrm{GHz}$ (solid line). The curves are presented of the angular range from -180 degrees to 180 degrees.

Fig. 5 shows the co-polar radiation patterns in the $E$ plane for the same frequencies: $4 \mathrm{GHz}$ (dashed line), 5 $\mathrm{GHz}$ (dotted line), $10 \mathrm{GHz}$ (solid line).

In both cases, the maximum of radiation occurs always in the same direction.

In Table I the $-3 \mathrm{~dB}$ angles in the $E$ and $H$ planes are compared. The variation of these angles with frequency is clearly slower in the $H$-plane where the radiation is dominated by the leakage than in the $E$-plane where it is dominated by the geometrical optics. In fact, at higher frequencies, the electric field on the slot is concentrated over a smaller part of the slot so that the directivity does not increase significantly with frequency in the $H$-plane as opposite with what happens in the $E$-plane.

\section{CONCLUSION}

In this work, we have designed a novel antenna topology, named the Pyramid antenna. It has unpaired bandwidth performances that can be briefly synthesized as follows:

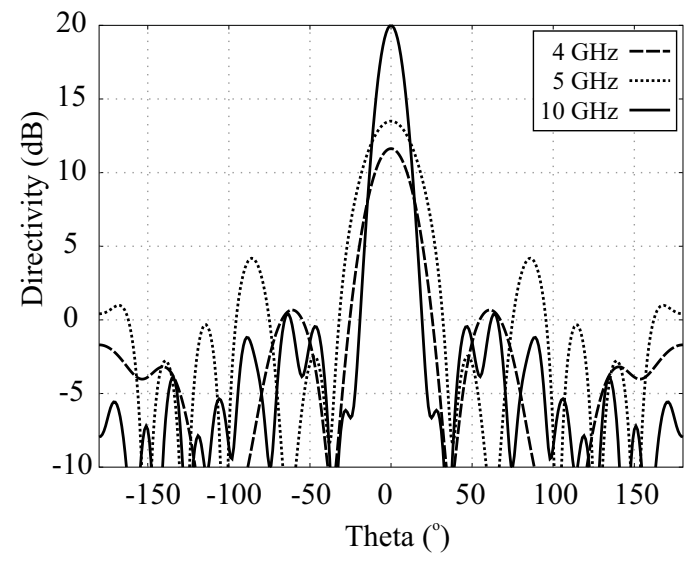

Fig. 4. Co-polar radiation pattern in the $H$-plane for three different frequencies: $4 \mathrm{GHz}$ (dashed line), $5 \mathrm{GHz}$ (dotted line), $10 \mathrm{GHz}$ (solid line). Simulations performed with HFSS.

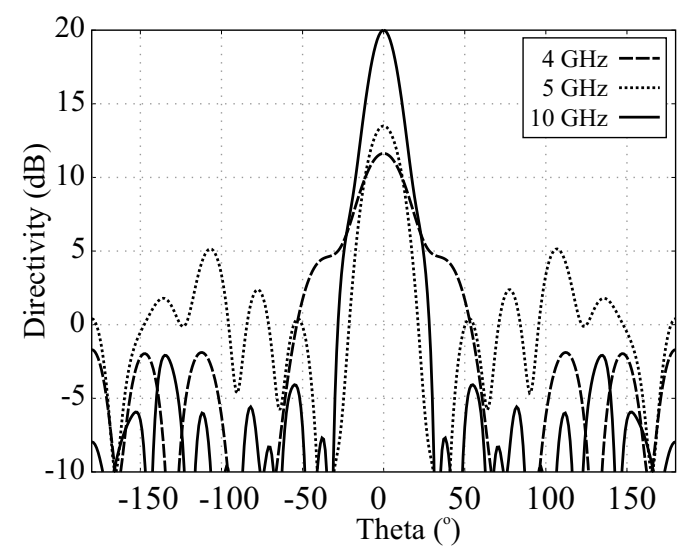

Fig. 5. Co-polar radiation pattern in the $E$-plane for three different frequencies: $4 \mathrm{GHz}$ (dashed line), $5 \mathrm{GHz}$ (dotted line), $10 \mathrm{GHz}$ (solid line).

1. Input impedance with limited frequency dependence. Reflection coefficients lower than -9dB's have been achieved over 1:10 bands.

2. Radiation pattern essentially frequency independent on the $H$-plane (on bands 1:10). In the $E$-plane the directivity grows as a result of the lens shaping.

3. With respect to all other broad band directive radiators the antenna's phase center is much more stable with frequency.

\section{REFERENCES}

[1] N. Kuwabara, K.Tajima, R. Kobayashi, F.Amemiya, "Development and analysis of electric field sensor using LiNbO3 optical modulator", IEEE Transaction Electromagnetic Compatibility, Vol 34, November 1992.

[2] Y.Tokano, H.Kobayashi, T.Miyakawa, Y.Houjyo, "A gigahertzrange micro optical electric field sensor", EMC Symposium, Zurich 2001.

[3] TNO Technical Note 200 "EM Design description" deliverable of the ESTEC contract no. ESTEC 16850/02/NL/LvH "UWB Integrated E.M. Field Sensor".

[4] A. Neto, S. Bruni, G. Gerini, M. Sabbadini, "The Leaky Lens: a Broad Band, Fixed Beam Leaky Wave Antenna", IEEE 


\begin{tabular}{|c|c|c|c|}
\hline $\begin{array}{c}\text { Frequency } \\
(\mathrm{GHz})\end{array}$ & $\begin{array}{c}\text { Directivity } \\
(\mathrm{dB})\end{array}$ & $\begin{array}{c}-3 \mathrm{~dB} \text { Angle } \\
H \text {-plane }\left(^{\circ}\right)\end{array}$ & $\begin{array}{c}-3 \mathrm{~dB} \text { Angle } \\
E \text {-plane }\left(^{\circ}\right)\end{array}$ \\
\hline 4 & 11.4 & 28 & 32 \\
\hline 5 & 13.24 & 26 & 22 \\
\hline 6 & 15.63 & 24 & 26 \\
\hline 7 & 15.9 & 18 & 24 \\
\hline 10 & 19.7 & 16 & 18 \\
\hline
\end{tabular}

TABLE I

Directivities AND -3 DB ANGLES IN THE $H$ AND $E$ PLANES FOR DIFFERENT FREQUENCIES.

Transactions on Antennas and Propagation, Vol. 53, no. 10, pp. 3240 - 3246, October 2005.

[5] S. Maci and A. Neto, "Green's function of an infinite slot line printed between two homogeneous dielectrics. Part II: Uniform asymptotic fields," IEEE Transactions on Antennas and Propagation, vol. 52, no. 3, pp. 666-676, March 2004. 